\title{
Towards a digital body: the virtual arm illusion
}

\author{
Mel Slater ${ }^{1,2,3, *}$, Daniel Perez-Marcos ${ }^{4,5}$, H. Henrik Ehrsson ${ }^{6}$ and Maria V. Sanchez-Vives ${ }^{1,4,5}$
}

1. Institució Catalana de Recerca i Estudis Avançats, Barcelona, Spain

2. Centre de Realitat Virtual, Universitat Politècnica de Catalunya, Barcelona, Spain

3. Department of Computer Science, UCL, London, UK

4. Instituto de Neurociencias de Alicante, Universidad Miguel Hernández-CSIC, Alicante, Spain

5. Institut d'Investigacions Biomèdiques August Pi i Sunyer, Barcelona, Spain

6. Department of Clinical Neuroscience, Karolinska Institute, Stockholm, Sweden

Edited by: $\quad$ Maurizio Corbetta, Washington University, USA

Reviewed by: Olaf Blanke, University of Geneva, Switzerland; Ecole Polytechnique Fédérale de Lausanne, Switzerland Nicholas Holmes, Hebrew University, Israel

\begin{abstract}
The integration of the human brain with computers is an interesting new area of applied neuroscience, where one application is replacement of a person's real body by a virtual representation. Here we demonstrate that a virtual limb can be made to feel part of your body if appropriate multisensory correlations are provided. We report an illusion that is invoked through tactile stimulation on a person's hidden real right hand with synchronous virtual visual stimulation on an aligned 3D stereo virtual arm projecting horizontally out of their shoulder. An experiment with 21 male participants showed displacement of ownership towards the virtual hand, as illustrated by questionnaire responses and proprioceptive drift. A control experiment with asynchronous tapping was carried out with a different set of 20 male participants who did not experience the illusion. After $5 \mathrm{~min}$ of stimulation the virtual arm rotated. Evidence suggests that the extent of the illusion was also correlated with the degree of muscle activity onset in the right arm as measured by EMG during this period that the arm was rotating, for the synchronous but not the asynchronous condition. A completely virtual object can therefore be experienced as part of one's self, which opens up the possibility that an entire virtual body could be felt as one's own in future virtual reality applications or online games, and be an invaluable tool for the understanding of the brain mechanisms underlying body ownership.
\end{abstract}

Keywords: rubber hand illusion, virtual reality, virtual environment

\section{INTRODUCTION}

Although daily experience suggests that our body image is highly stable there is strong experimental evidence that demonstrates that in fact it is quite malleable. Synchronous tactile stimulation (tapping and stroking) on a visible rubber arm placed in front of a person and on their hidden real arm results, under certain conditions, in displacement of sensation of feeling towards and an illusion of ownership of - the fake arm (Botvinick and Cohen, 1998). This is usually called the 'rubber hand illusion'. The experiments have been replicated several times. For example Armel and Ramachandran (2003) have shown that skin conductance responses were associated with manipulations of the rubber hand that would normally cause pain. These authors also reported that participants could experience a weaker illusion for objects that may appear to be nothing like an arm - such as a table top that was synchronously tapped with the real hand.

*Correspondence: Mel Slater, ICREA-Centre de Realitat Virtual (CRV), Edificio U, Universitat Politècnica de Catalunya, C. Pau Gargallo 16, 08028 Barcelona, Spain. e-mail:m.slater@cs.ucl.ac.uk

Received: 10 January 2008; paper pending published: 16 March 2008; accepted: 07 July 2008; published online: 20 August 2008.

Citation: Front. Hum. Neurosci. (2008) 2: 6. doi: 10.3389/neuro.09.006.2008

Copyright () 2008 Slater, Perez-Marcos, Ehrsson and Sanchez-Vives. This is an openaccess article subject to an exclusive license agreement between the authors and the Frontiers Research Foundation, which permits unrestricted use, distribution, and reproduction in any medium, provided the original authors and source are credited.
This latter result has been called into question by Tsakiris and Haggard (2005), who showed, amongst other things, that for the illusion to work at all it is required that the fake arm look like an arm, and that the rubber arm should be aligned with the orientation of the real arm (see also Pavani et al., 2000; Ehrsson et al., 2004 for similar observations). There have also been results from a brain imaging study by Ehrsson et al. (2004) who identified activity in specific multisensory brain areas associated with the illusion, and that this activity was greater when the rubber arm was aligned in parallel with the real one. Regarding the underlying mechanisms it has been suggested that the illusion occurs as the result of the integration and interpretation of visual, tactile and position sense (proprioceptive) signals (Botvinick and Cohen, 1998; Ehrsson et al., 2004). Neurons in multisensory areas that integrate this information could implement the necessary neuronal computations causing changes in body ownership (Ehrsson et al., 2004, 2005).

In this study we show that the illusion can be produced within an immersive virtual environment (VE), and provide subjective, behavioural and physiological evidence for this. An immersive $\mathrm{VE}$ is one that in principle entirely replaces real sensory data by computer generated data. Participants in such an environment are also head-tracked so that the virtual scene is displayed as a function of head position and orientation. Overall, participants are likely to respond realistically to the virtual scene (SanchezVives and Slater, 2005). In a VE the participants could have a 
virtual body, with additional limbs, for example, controlled by a brain-computer interface (BCI). Is it possible to make virtual limbs feel like the person's own? This may seem like science fiction but current experiments point strongly to these possibilities. Controlling the virtual world with $\mathrm{BCI}$ has been demonstrated (Pfurtscheller et al., 2006). Here we concentrate on ownership of a virtual limb.

\section{MATERIALS AND METHODS VIRTUAL REALITY SYSTEM}

The particular virtual reality system that we used ${ }^{1}$ consisted of a $2 \mathrm{~m} \times 2.7 \mathrm{~m}$ display on which computer generated images are back-projected from two BenQ projectors. The projectors are fitted with polarising lenses, and correspondingly the participant wears glasses with polarising lenses. The computer generates both left eye and right eye images which are filtered by the polarising lenses so that passive stereo is realised. An Intersense 900 tracking system is used which has the capability for six degrees of freedom tracking (position and orientation) of two devices - one is located on the participant's head and the other is for a hand-held 'Wand' device that has five buttons with which to effect interactions.

\section{RECRUITMENT AND PROCEDURES}

Twenty-one healthy male participants (mean age and standard deviation $22 \pm 3.9$ years) were recruited for the experiment by advertisement amongst students, research and administrative staff at the Instituto de Neurociencias, UMH. They were each offered $5 €$ for their participation. Upon arrival at the laboratory they were asked to read and sign a consent form, the experiment having been carried out in accordance with the regulations of Comité Ético de Investigación del Hospital Universitario de San Juan de Alicante, Spain. Each participant stood beside a mounted shoulder-high wooden shelf (adjustable in height) and rested his right arm on the shelf. His arm was hidden from his view by a sheet of grey foam fixed to the side of the shelf. The participant wore the stereo glasses and the head-tracker mounted just above the glasses (Figure 1A).

The computer program generated a stereo image of a virtual arm (Figure 1B). The experimenter could use the Wand device to interactively position the arm to a place that the participant reported as being correct, and also could interactively adjust the scale of the arm so that it appeared to be the right size. From the participant's point of view the arm appeared to project out of his right shoulder - almost parallel to the real one. However, unlike the real arm, the virtual arm was not shown resting on a shelf, but held outwards in front with the distance between the participant's real hand and the virtual hand approximately $20 \mathrm{~cm}$. Due to the head tracking, were the participant to sway his body from side to side, then the arm would move appearing to be attached to the person's body. If the participant kept his body still and just moved his head as if to look at the arm from a different position, then the arm would appear to be stationary, as it would in reality. This was achieved by fixing the arm relative to a fixed position on the neck unaffected by head rotations. The setup was, therefore, able to powerfully induce the illusion that there was an arm pointing straight ahead, which appeared to be attached to the participant's body in the usual way.

${ }^{1}$ VRmedia - www.vrmedia.it.

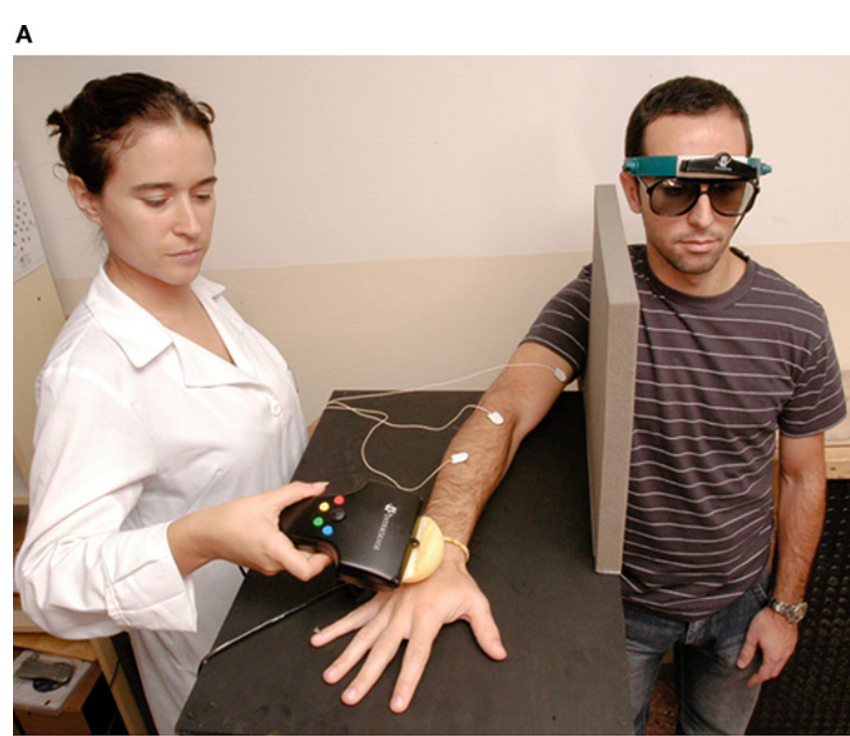

B

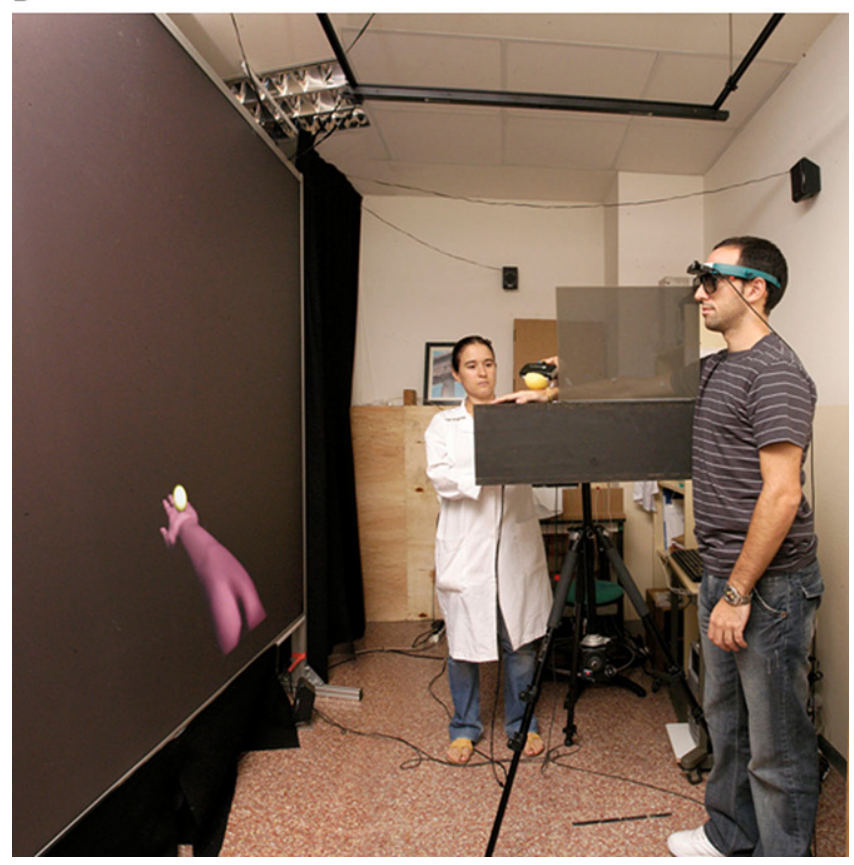

Figure 1 | The experimental setup. (A)The participant wears passive stereo glasses and a head-tracker, and the virtual image is determined as a function of his head direction. The experimenter taps and strokes the participant's real hand with a 6-degree freedom Wand, whose position is tracked and used to determine the position of the virtual sphere. $(\boldsymbol{B})$ The participant is standing in front of a $2 \mathrm{~m} \times 2.7 \mathrm{~m}$ rear projection screen. The arm in the screen is seen from the participant's point of view as projecting out of his right shoulder, while his own arm is out of view and resting on a support. In the projection the participant also sees a sphere striking in synchrony and in the same place on the virtual hand as the touch stimuli delivered to his own hand

Prior to being fitted with the virtual reality equipment, but when already standing next to the shelf with his arm resting on it, the participant was given a piece of blue-tack in his left hand, and invited to place it under the shelf in a position corresponding to where he felt the centre of his right palm to be. Then the virtual arm was displayed and interactively positioned and scaled until the participant reported that it appeared to be correct, and then fixed in position relative to the neck. The position of the Wand 
device was then represented by a small virtual ball. Therefore, as the experimenter moved the Wand, the virtual sphere would move correspondingly. The experimenter stood on the other side of the shelf and the gray foam to the participant (Figure 1A). A soft ball had been fixed to the base of the Wand. When the experimenter touched the participant's real hand with this ball the virtual ball would appear to touch the virtual hand in the same position (Figure 1). Thus, from the point of view of the participant, he would feel his hand being tapped and stroked by the physical ball, but see a virtual ball that similarly and synchronously touched and stroked the virtual hand that was projecting out of his real body. All the lights in the room were switched off, and the only illumination was from the projection of the virtual arm itself.

The tapping and stroking continued for $5 \mathrm{~min}$, with an attempt made to make the stimulation irregular and unpredictable. At the end of this period the tapping stopped, and the virtual arm slowly rotated by $90^{\circ}$ so that the palm of the hand would become orthogonal to the ground plane (supination), and then rotated back again (pronation), the whole sequence lasting $12 \mathrm{~s}$. After this, a message appeared on the screen inviting the participant to close his eyes, and again quickly place a second piece of blue-tack where he felt his right hand to be. This allowed the measurement of the 'proprioceptive drift' - the difference between the originally felt place of the hand and the place of the hand after the experiment. In this measurement, only the horizontal displacement was taken into account, discounting displacements that could be related to feeling the arm shorter or longer. A similar approach was used by Botvinick and Cohen (1998) to provide behavioural evidence for the original rubber hand illusion.

One bipolar EMG (electromyogram) channel was recorded from the right arm of each participant. Each subject was fitted with three surface electrodes, two on the supinator muscle (about $2 \mathrm{~cm}$ spacing) and the ground on the biceps. The voltage signal was fed on gUSBamp amplifiers (Guger Technologies, Graz, Austria) with a sampling rate of $256 \mathrm{~Hz}$. The purpose of this was to measure the degree of activity in the forearm muscles throughout the period of the experiment, in order to test for differences between activity during the last $12 \mathrm{~s}$ when the virtual arm rotated and the earlier 5-min period when the virtual arm was still. There were 20 usable EMG recordings obtained, one recording being of poor quality.

After the conclusion of the experiment the participants were invited to complete a 9-item questionnaire. Questions were taken from Botvinick and Cohen (1998), translated into Spanish. The questions were presented in a different randomised order for each participant following the method introduced by Botvinick and Cohen (1998). Each question was rated by the participants using a 7-point Likert scale, with 1 meaning 'totally disagree' and 7 'totally agree'. Questions 1, 2 and 3 are supposed to indicate the illusion, and the remaining questions 4-9 are usually considered as control questions. The questions were:

1. Sometimes I had the feeling that I was receiving the hits in the location of the virtual arm.

2. During the experiment there were moments in which it seemed as if what I was feeling was caused by the yellow ball that I was seeing on the screen.

3. During the experiment there were moments in which I felt as if the virtual arm was my own arm.

4. During the experiments there were moments in which it seemed that my real arm was being displaced towards the left (towards the virtual arm).
5. During the experiment there were moments in which it seemed that the contact that I was feeling originated in some place in between my own arm and the virtual arm.

6. During the experiment there were moments in which I felt as if my real arm was becoming virtual.

7. During the experiment there were moments in which it seemed (visually) that the virtual arm was being displaced towards the right (towards my real arm).

8. During the experiment there were moments in which the virtual arm started to look like my own arm in some aspects.

9. During the experiment there were moments in which I had the sensation of having more than one right arm.

An asynchronous condition was carried out with a different set of 20 males (mean age and standard deviation $32 \pm 6.3$ ) where the visual tapping did not correspond with the tactile tapping. The tracking data from one real session of tapping and stroking was recorded, and this was used to generate the visual tapping for all of the control condition trials. Therefore the visual tapping and stroking would look visually plausible, but would not correspond to the tactile stimulation experienced by any of the participants. The EMG was recorded at $1200 \mathrm{~Hz}$, and there were 19 usable datasets.

\section{EMG ANALYSIS}

The EMG analysis compared the number of activity onsets during the time that the virtual arm was seen as still to the time that it was rotating based on the method described by Di Fabio (1987) (see a further review by Hodges and Bui, 1996). In this method a $50-\mathrm{Hz}$ low pass filter is applied to the rectified signal and all further computations are on this rectified filtered signal. The standard deviation $(\sigma)$ of a $50-\mathrm{ms}$ period of the signal before activity onset is computed. Onset activity is defined as a portion of the subsequent signal that has amplitude of at least $3 \sigma$ for at least $25 \mathrm{~ms}$. Since this method was used to detect onset activity in conditions where the subjects were told to deliberately make a muscle contraction, our method is slightly different, since we did not know whether or not there would be any detectable activity, and in any case such activity would be likely to be involuntary (induced by observation of virtual arm rotating). Therefore we computed the standard deviation $\sigma$ over the middle period of this data (100-200 s), treating the first third of the data as 'settling in' time). Then we computed the number of onsets in the last third of the signal (between 200 and $300 \mathrm{~s}$ ), and also computed the number of onsets during the time the virtual arm was rotating. Due to the sampling frequencies that we had available, we took the period indicating an activity onset to be $(1 / 32)$ seconds $(31.25 \mathrm{~ms})$ in the synchronous case, and $(1 / 40)$ seconds $(25 \mathrm{~ms})$ in the asynchronous case (Varying these timings does not alter the results presented below). For example, an activity onset in the synchronous case is defined to be a portion of the signal with values at least three times the standard deviation that lasts for at least $1 / 32 \mathrm{~s}$.

\section{RESULTS}

Our major hypothesis was that synchronous tactile stimulation of the person's real hand and the entirely virtual one would result in the same illusion as that which has previously been reported for the rubber hand illusion. This would be shown first by significantly higher responses for questions 1,2 and 3 compared to questions 4-9. Second, the drift measurement of the perceived hand position should show average drift significantly higher than zero. We expected that the illusion would not be shown 
in the asynchronous control condition, and therefore we predicted greater illusion-question-scores and proprioceptive drift in the synchronous condition as compared to the asynchronous condition. Regarding EMG we expected that in the synchronous condition the muscle activity would be shown to be greater in the last $12 \mathrm{~s}$ (while the virtual arm rotated) than during the $100 \mathrm{~s}$ before the rotation, but that this would not be the case for the asynchronous condition. We also expected the EMG activity to be correlated with the extent of the subjective illusion in the synchronous condition.

\section{QUESTIONNAIRE RATINGS}

The boxplots for the questionnaire results are shown in Figure 2. For the synchronous condition Q1-Q3 have higher scores than the remainder. For the asynchronous condition the results for Q1-Q3 are not significantly different from the remainder, and all the scores are low.

In order to compare results across the questions within each condition we first carried out a Kruskal-Wallis non-parametric one-way analysis of variance. This tests the null hypothesis of equal medians across all of the questions. In the case of the synchronous condition this hypothesis is rejected in favour of the alternative hypothesis that not all medians are equal $\left(P=3.3 \times 10^{-9}\right)$. In order to compare the medians with one another we use the Tukey-Kramer multiple comparisons test, which takes account of possible correlations between the scores of the different questions. Using an overall significance level of $5 \%$ we find that (with respect to the medians)

$$
\begin{aligned}
& \text { Q1 > Q4, Q5, Q7, Q9 } \\
& \text { Q2 > Q4, Q5, Q6, Q7, Q9 } \\
& \text { Q3 > Q4, Q5, Q7, Q9 }
\end{aligned}
$$

where ' $>$ ' means that the median of the variable on the left is significantly greater than all those on the right. Amongst the control questions the median of Q6 is not significantly less than that of Q1 and Q3, and the median of Q8 is not significantly less than any of Q1-Q3. We discuss this further in section Questionnaire Issues.
If we carry out the same analysis on the asynchronous questionnaire results then we find that the null hypothesis of equal medians across all questions is not rejected $(P=0.70)$.

In addition we analysed the questionnaire results very conservatively by only considering the high scores (' 6 ' or ' 7 ') for each question as indicating the illusion, in other words transforming the 7-point questionnaire responses into a binary response ('high score' or 'not high score'). The scientific motivation for this is that we wanted to run an analysis which only takes into account people who feel a really strong illusion, making sure that our significant effects were psychologically relevant and comparable to the original rubber hand illusion which is many cases very vivid. By chance alone, the probability of a high score is $2 / 7$. In the case of the synchronous condition the answers to questions 1,2 and 3 have frequencies that are very much higher than would be expected by chance $(14,17$ and 12 respectively out of $n=21$, with corresponding $P$-values $0.0003,0.0000$ and 0.0057 , using the binomial distribution). The remaining questions are in accord with chance (the $P$-values ranging from 0.1157 to 0.9920$)$. In the case of the asynchronous condition the number of high responses to questions 1, 2 and 3 were 0,1 and 3 respectively out of $n=20$ (which are actually lower than would be expected by chance).

Finally, a comparison between the scores on the synchronous and asynchronous conditions is given in Table 1. It can be seen for the three questions that indicate the illusion that the median scores are significantly higher for the synchronous condition compared to the asynchronous condition. However, they are also higher for Q5, Q6 and Q8 (see Discussion).

\section{PROPRIOCEPTIVE DRIFT}

We observed a significant proprioceptive drift only in the synchronous condition (see below), and importantly this drift was greater than in the asynchronous control group.

For 18 of the 21 participants in the synchronous condition we have the measure of proprioceptive drift (D), which is the signed horizontal distance between their originally located hand position as referenced by the blue-tack, and the position indicated at the
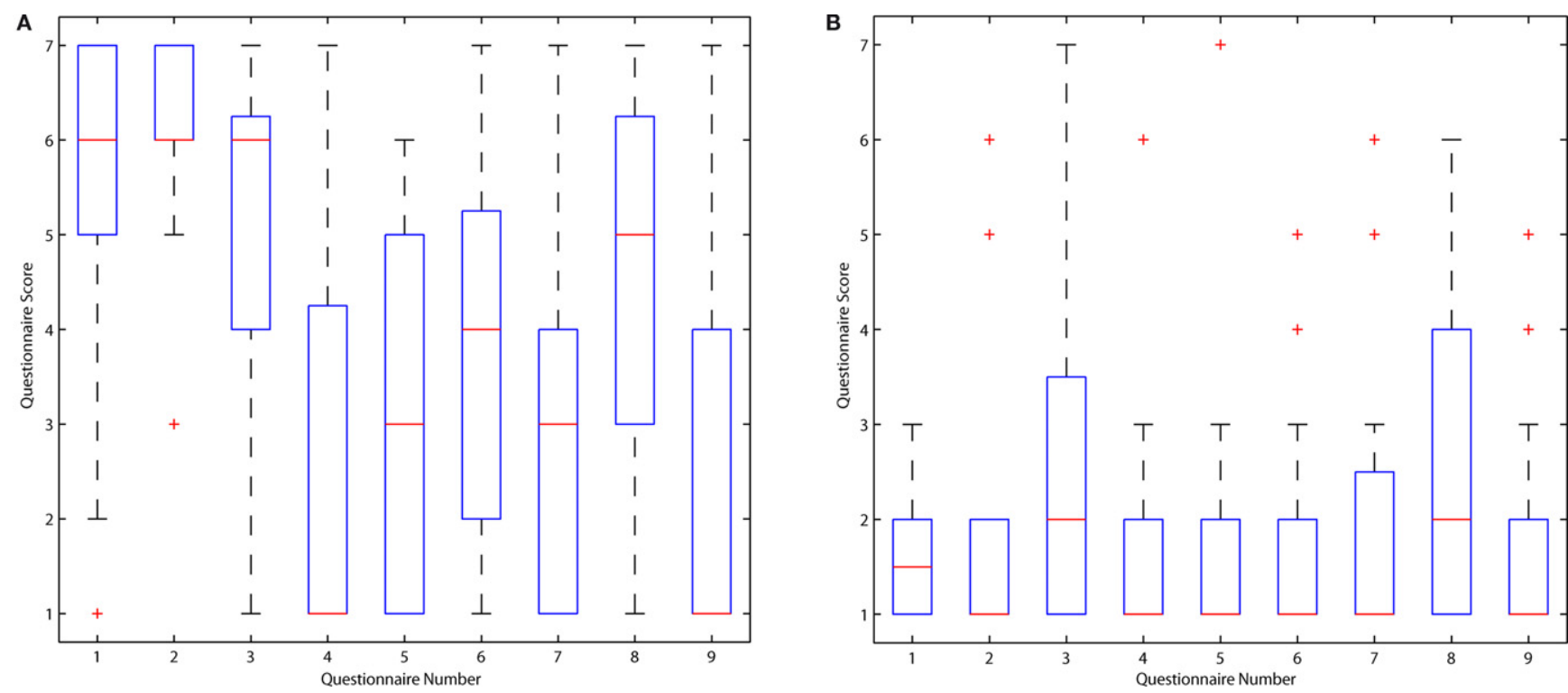

Figure 2 | Boxplots for the questionnaire responses in the two conditions. (A) Synchronous condition. (B) Asynchronous condition. Questions 1, 2 and 3 address the illusory experience. The medians are shown as red lines, and the boxes are the interquartile ranges (IQR). The whiskers represent either the extreme data points or extend to $1.5 \times$ IQR. If there are values outside the whiskers these are conventionally called 'outliers', and are shown by $(+)$. 
Table 1 | Medians and interquartile ranges of the questionnaire scores.

\begin{tabular}{llllllllll}
\hline & $\mathbf{Q 1}$ & $\mathbf{Q 2}$ & $\mathbf{Q 3}$ & $\mathbf{Q 4}$ & $\mathbf{Q 5}$ & $\mathbf{Q 6}$ & $\mathbf{Q 7}$ & $\mathbf{Q 8}$ & $\mathbf{Q 9}$ \\
\hline Synchronous $(n=21)$ & $6(2)$ & $6(1)$ & $6(2.25)$ & $1(3.25)$ & $3(4)$ & $4(3.25)$ & $3(3)$ & $5(3.25)$ & $1(3)$ \\
Asynchronous $(n=20)$ & $1.5(1)$ & $1(1)$ & $2(2.5)$ & $1(1)$ & $1(1)$ & $1(1)$ & $1(1.5)$ & $2(3)$ & $1(1)$ \\
$P$ & 0.0000 & 0.0000 & 0.0002 & 0.2202 & 0.0083 & 0.0005 & 0.0893 & 0.0006 & 0.4491 \\
\hline
\end{tabular}

This table shows the medians and interquartile ranges (in brackets) of the questionnaire scores, and the $P$ values indicate the results of the rank sum (Mann-Whitney U) test, which tests the hypothesis that the scores from the synchronous and asynchronous groups come from distributions with equal medians.
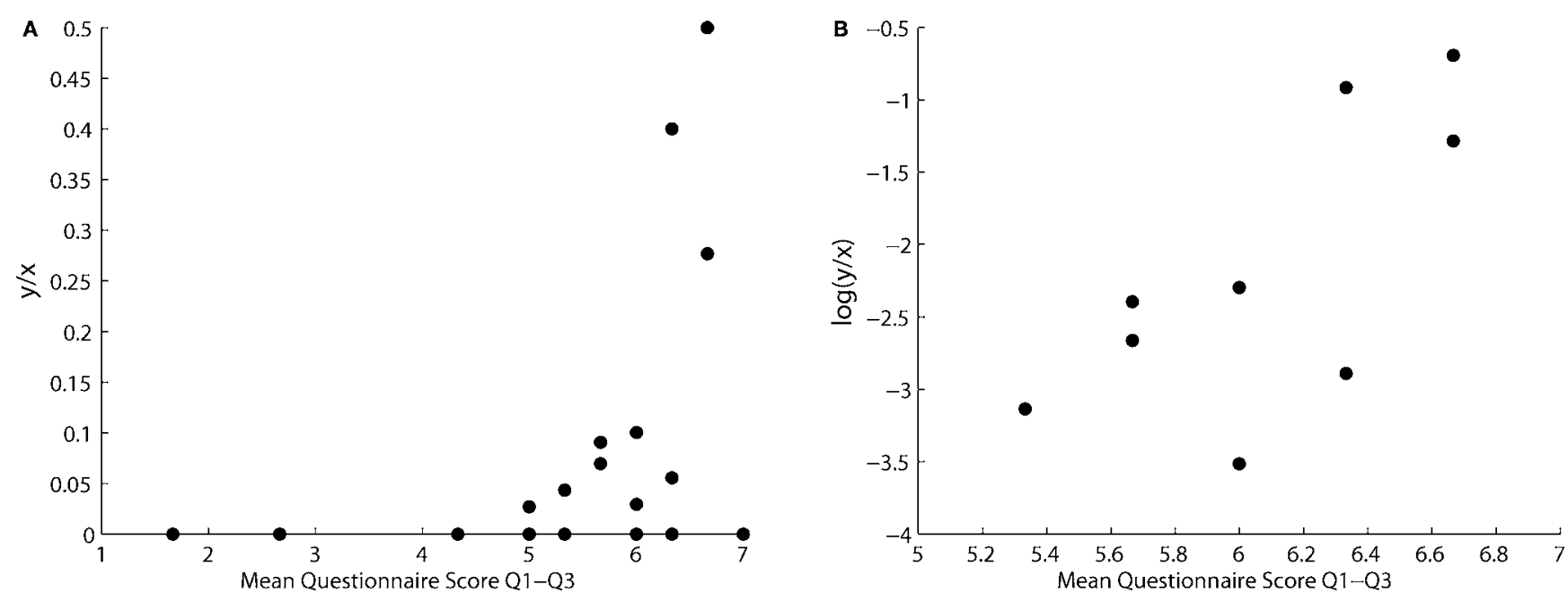

Figure 3 | Relationship between EMG activity and subjective illusion ratings. Scatterplot of $y_{t} / x$ and $\log \left(y_{t} / x\right)$ on the mean of questions Q1-Q3 ( $\left.q_{\text {mean }}\right)$, for $t=6$. $y$ is the number of activity onsets during the first $t=6 \mathrm{~s}$ that the virtual arm was rotating, $x$ is the number of activity onsets in the $100 \mathrm{~s}$ prior to the virtual arm rotating. (A) $y_{t} / x$ by $q_{\text {mean }}$ over all observations, (B) $\log \left(y_{t} / x\right)$ by $q_{\text {mean }}$ for observations with $y_{t}>0$.

end of the experiment. The distance is signed as positive if it was on the side of the hand towards the virtual hand, and negative if on the other side. Higher positive drift indicates a greater recalibration of position sense by the illusion. The range of $\mathrm{D}$ was from -15 to $130 \mathrm{~mm}$, with median $30 \mathrm{~mm}$. A non-parametric sign test rejects the hypothesis that the median is zero $(P=0.0063)$. The three missing data were due to the original blue-tack falling off, or the participant not following the instructions correctly.

For the asynchronous condition D ranged from -50 to $35 \mathrm{~mm}$, with median 0 , and the sign test does not reject the hypothesis of 0 median. A rank sum (Mann-Whitney $U$ ) test rejects the hypothesis that the two sets of $\mathrm{D}$ values are from distributions with equal median $(P=0.0026)$. In other words the drift was greater in the synchronous condition than in the asynchronous condition.

\section{EMG ANALYSIS}

Our hypotheses regarding the EMG were that (1) the mean number of activity onsets over all participants would be greater during the time that the virtual arm was rotating compared to the time before the arm rotated. The number of activity onsets would (2) show a positive correlation with the strength of the illusion as measured by the questionnaire (specifically the mean of Q1-Q3). We expected that (1) and (2) would be true for the synchronous but not the asynchronous condition.

Let $y_{i t}$ be the number of activity onsets (see EMG Analysis) in the first $t$ seconds of arm rotation for participant $i$, and $x_{i}$ the number in the period ( $T=100 \mathrm{~s}$ ) before the arm rotation for participant $i$. The variable $x_{i}$ is a baseline measure of the number of activity onsets for participant $i$.
Let $q_{i \text { mean }}$ be the mean response of Q1, Q2 and Q3 for participant $i$. (In what follows we drop the subscript $i$ to improve readability). Since $y_{t}$ represents the number of occurrences of an event (the onsets) randomly distributed through time it should follow a Poisson distribution. Of interest will be the ratio of $y_{t} / t$ to $x / T$, and how this ratio varies with $q_{\text {mean }}$. We can carry out a standard log-linear regression of $y_{t} / x$ on $q_{\text {mean }}$ as the explanatory variable of interest.

The regression model is:

$\log \left(\frac{E\left(y_{t}\right) / t}{x / T}\right)=\beta_{0}+\beta_{1} q_{\text {mean }}$

or

$\log \left(E\left(y_{t}\right)\right)=\log \left(\frac{x \cdot t}{T}\right)+\beta_{0}+\beta_{1} q_{\text {mean }}$

This is a log-linear model, a special case of a generalized linear model with a Poisson distribution, log link function, and offset $\log (x \cdot t / T)$, where $y_{t}$ has a Poisson distribution with expected value $E\left(y_{t}\right)$ (McCullagh and Nelder, 1989). In our model fitting procedure we relax the strict requirement that $y_{t}$ has a Poisson distribution and allow for over-dispersion (Breslow, 1984).

Our hypotheses can be stated in terms of this model as (1) $\beta_{0}>0$ and (2) $\beta_{1}>0$ where the null hypotheses are that $\beta_{j}=0$ $(j=0,1)$. The null hypothesis for $(1)$ is not rejected, and the null hypothesis for (2) is rejected. The latter result suggests that the sensory illusion is somehow recruiting the motor system.

Figure $3 \mathrm{~A}$ shows the plot of all $y_{t} / x$ by $q_{\text {mean }}$, for $t=6 \mathrm{~s}$, which is the time by which the arm had fully rotated to its new position 
Table 2 | Significance levels and correlation coefficients for the regression coefficient of the log-linear regression Eq. (1).

\begin{tabular}{|c|c|c|c|c|c|c|c|c|c|c|}
\hline \multirow[b]{2}{*}{$t$} & \multicolumn{5}{|c|}{ Synchronous } & \multicolumn{5}{|c|}{ Asynchronous } \\
\hline & $\beta_{1}$ & $P\left(\beta_{1}\right)$ & $r$ & $P$ & $n$ & $\beta_{1}$ & $P\left(\beta_{1}\right)$ & $r$ & $P$ & $n$ \\
\hline 2 & 1.2 & 0.084 & 0.54 & 0.209 & 7 & 0.0 & 0.980 & 0.00 & 0.993 & 17 \\
\hline 3 & 1.0 & 0.048 & 0.61 & 0.107 & 8 & 0.1 & 0.778 & 0.01 & 0.974 & 17 \\
\hline 4 & 1.2 & 0.021 & 0.69 & 0.039 & 9 & 0.1 & 0.760 & 0.06 & 0.829 & 17 \\
\hline 5 & 1.1 & 0.015 & 0.71 & 0.022 & 10 & 0.0 & 0.945 & 0.02 & 0.933 & 17 \\
\hline 6 & 1.1 & 0.011 & 0.77 & 0.010 & 10 & 0.0 & 0.904 & 0.03 & 0.902 & 17 \\
\hline
\end{tabular}

$t$ is the number of seconds after the virtual arm started rotating, $\beta_{1}$ is the coefficient in $E q .(1)$ and $P\left(\beta_{1}\right)$ is the corresponding significance level, $r$ is the normal correlation coefficient between $\log \left(y_{t}, x\right)$ and $q_{\text {mean }}$, for entries with $y_{t}>0, P$ is the significance level for $r, n$ is the number of entries on which $r$ is based (all those for which $y_{t}>0$ ).

before rotating back. In Figure 3B the same data is shown with all values with $y_{t}=0$ eliminated, in case these were thought to be biasing the result, and on a log scale for the vertical axis. In each case it can be seen that there is a strong relationship between the two variables.

In Table 2 we show the results for all $t$ between 2 and $6 \mathrm{~s}$ for synchronous and asynchronous conditions. It can be seen that between 4 and $6 \mathrm{~s}$ after the arm started moving there is a significant positive association between the number of onsets and the strength of subjective illusion in the synchronous but not in the asynchronous condition (after accounting for the baseline values, $x$ ). The table also shows the correlation coefficients between $\log \left(y_{t} / x\right)$ and $q_{\text {mean }}$, for entries with $y_{t}>0$, to take account of the possibility that the zero entries for $y_{t}$ might have biased the results of the regression analysis.

If we carry out an analysis using the mean of the questions that are not supposed to indicate the illusion (Q4-Q9) then we find nothing to be significant. For example, for the synchronous condition the minimum $P(\beta 1)$ corresponding to Table 2 is 0.50 and the minimum $P$ is 0.26 . The same is true for the asynchronous condition [minimum $P(\beta 1)$ and $P$ are 0.55 and 0.56 respectively].

\section{DISCUSSION \\ MAIN FINDINGS}

The experiment reported here demonstrates that the 'rubber hand illusion' can be replicated with an entirely virtual threedimensional arm and hand - not only is the arm virtual, but the ball that is seen to strike it is virtual too. The results are comparable with the original Botvinick and Cohen experiment with respect to the subjective reporting of the illusion and with respect to the proprioceptive drift, with both these measures being greater in the synchronous condition than in the asynchronous condition. Second, we found a positive correlation between the reported subjective strength of the illusion and the number of EMG activity onsets while the virtual arm was rotating. These results did not occur for the asynchronous condition. Moreover there was no association between the non-illusion questions and this EMG measure in any condition. Although we did not find a greater mean number of EMG onset activity while the arm was moving compared to when it was not moving, we would emphasise that the EMG results are indicative of a possible relationship and further experimentation is required.

\section{QUESTIONNAIRE ISSUES}

The results are tempered by two issues: the first is that the questionnaire scores for some of the non-illusion questions are significantly greater for the synchronous condition than for the asynchronous condition (Table 1). A priori there is no reason why participants who felt the illusion of ownership to some extent might not also give relatively high scores to these questions: a confusion about the location of the felt touch (Q5), that their arm was virtual (indeed it would be so!) (Q6) and a feeling of change of appearance of the virtual arm to become like their own arm (Q8). This is partly also a problem of the use of questionnaires in general to elicit such subjective phenomena. It has been shown for example that it is quite easy to obtain seemingly meaningful questionnaire responses based on an entirely invented feeling simply by asking questions about it (Slater, 2004), so that over-reliance on questionnaires is methodologically dubious. It should be noted also in this context that the pattern of responses to the questions in the synchronous condition in terms of location and dispersion is almost identical to that in Botvinick and Cohen (1998), though we do not have information from that experiment about a question-by-question comparison of the synchronous and control conditions.

In fact for the synchronous condition the means of the illusion questions and non-illusion questions are correlated $(r=0.52$, $P=0.02)$, and similarly for the asynchronous condition $(r=0.77$, $P=0.0001)$. This strongly illustrates that participant's responses to the illusion are captured by their responses to the entire questionnaire rather than simply to the designated three questions, and that further reliance on this particular questionnaire alone would be not be appropriate. The more important finding in the present study is the significant difference in scores between the synchronous and asynchronous conditions on questions 1-3.

\section{PROPRIOCEPTIVE DRIFT ISSUES}

The significant shift in the felt location of the right arm towards the virtual arm (proprioceptive drift) after a period of synchronous stimulation was an important objective measure of the illusion, a drift that did not occur in the case of asynchronous stimulation. In both cases the original piece of blue-tack placed before the experiment remained in position, so it is possible that participants could search to find that piece and then choose their second position based on this. However, this does not reduce the validity of the results for three reasons. First, participants were asked to quickly place the piece of blue-tack so that there would not be time to find the original piece. Second, if anything, this would introduce a bias that would tend to reduce the amount of drift - through participants placing the second piece next to where they might have felt the first piece. Third, in any case the conditions were the same for both the synchronous and asynchronous groups, and yet the results are significantly different. 
One further point with respect to drift is that in rubber arm illusion experiments the drift is determined immediately after the completion of the tapping and stroking. In our case there was a gap of $12 \mathrm{~s}$ while the arm rotated before the instruction was given to place the second piece of blue-tack. This gap should, if it has any effect at all, diminish the effect of the synchronous tapping, reducing the chance of observing proprioceptive drift.

\section{PILOT EXPERIMENTS}

It should be noted that the particular setup that we used was arrived at after pilot studies with about 30 participants using different configurations that either did not result in the illusion, or in which the illusion was reported to a much lesser extent. For example, when the participants would be seated the projection of the virtual arm would be cut off at the lower boundary of the display with our particular virtual reality system, so that the stereo projection would not reach all the way to the shoulder of the participant - hence it would look as if there would be a gap between the start of the virtual arm and real shoulder. In this condition the illusion did not occur. Second, in another condition we had the participants standing with their arm hanging loosely at the side and the virtual arm pointing out to the front. This again did not result in an experience of the illusion for most participants, but there was a marked degree of reporting of the sensation of tiredness in the right arm after the experiment (even though their real right arm had actually been hanging at the side). This also supports the earlier reported results that the rubber and real hand should be similarly oriented (Ehrsson et al., 2004; Tsakiris and Haggard, 2005). Also good registration between the position where the hand was tapped and stroked and the corresponding visual appearance of this with the virtual hand and ball was essential - if the hand appeared to be tapped in one place, but the visual appearance showed the ball to be tapping the virtual hand in another place, then the illusion did not work. Similarly, the coordinates of the virtual ball touching the virtual hand had to be adjusted finely such that the ball would not 'sink' deep into the hand, which would as well disrupt the illusion.

\section{BODY ILLUSIONS WITH VIRTUAL REALITY}

There have been several studies that have used something akin to virtual reality demonstrating the rubber hand illusion. Ijsselsteijn et al. (2006) used a video projection of a hand on a table top, though the strength of the illusion was significantly lower compared to the use of a physical rubber arm, as was the case for the proprioceptive drift. For example, Figure 2 of that paper shows that the mean scores for Q1-Q3 in their video projection condition to be lower than for their physical rubber arm condition. In (Durgin et al., 2007) it was shown that a version of the illusion can also be invoked by stroking a fake hand with a bright beam of light. Here subjects reported feeling tactile and thermal sensations associated with the light, and also ownership towards the fake hand comparable to the rubber hand illusion.

Beyond the projection of just an arm other recent studies have shown that people can recognize a mannequin as themselves (Lenggenhager et al., 2007) when looking at it through head-mounted displays connected to video-cameras. Similarly, people can be made to feel an illusion of being in different place from their seen physical body and lose the sense of ownership of it using a similar video-based technique (Ehrsson, 2007).

The present experiment is different because it demonstrates the feasibility of inducing a feeling of ownership of simulated body parts in a virtual environment, rather than people looking at part or all of their real selves, or other physical objects, whether using a projector or a head-mounted display with a mounted video camera. Thus unlike the previous experiments when real world objects in physical reality become part of the body, the present experiment shows that a simulated object can be fully incorporated into the body representation and become part of the participant. This phenomenon opens the doors to experiments in virtual reality that are impractical or impossible in the physical world, including real-time modifications of the virtual limb (length, size, appearance) and complex motions.

\section{CONCLUSIONS}

Virtual reality offers the possibility for a highly flexible approach to the problem of body image and self representation, and the conditions under which it can be transformed. Here we have exploited one of the possibilities opened up through the use of virtual reality, for example, the movement of the virtual arm, something that would be difficult to achieve systematically with the conventional rubber arm. The effect of this virtual movement on the behaviour (eventual movement of the real arm) or physiological measures (EMG) of the participant allows a wider objective evaluation of the illusion. This experiment is a first step in a set of studies that now intend to exploit its power in investigation of this phenomenon.

For the future our work also suggests that people can have their 'self' enter the virtual domain in a genuine sense of the word, and not just metaphorically as in current day computer games and online communities. In combination with BCI we envisage a functioning virtual body that is felt as their own by participants, with significant applications in VR training, limb prosthetics, and entertainment.

\section{SUPPLEMENTARY MATERIAL}

The Supplementary Material for this article can be found online at http://www.frontiersin.org/humanneuroscience/paper/10.3389/ neuro.09/006.2008/.

\section{CONFLICT OF INTEREST STATEMENT}

The authors declare that the research was conducted in the absence of any commercial or financial relationships that could be construed as a potential conflict of interest.

\section{ACKNOWLEDGEMENTS}

This work is funded under the European Union FET project PRESENCCIA Contract Number 27731. Support was also obtained from the Spanish Ministry of Science and Innovation. HHE was also supported by the Swedish Foundation for Strategic Research, the Swedish Medical Research Council, and the Human Frontier Science Program. We would like to thank Franco Tecchia and Marcello Carrozzino for their help with the XVR programming system. We thank Vanessa FernandezDescalzo, Carlos Quesada and Juan Francés for their participation in the realisation of the pilot experiments.

\section{REFERENCES}

Armel, K. C., and Ramachandran, V. S. (2003). Projecting sensations to external objects: evidence from skin conductance response. Proc. R. Soc. Lond. B. Biol. Sci. 270, 1499-1506.

Botvinick, M., and Cohen, J. (1998). Rubber hands 'feel' touch that eyes see. Nature 391, 756. 
Breslow, N. (1984). Extra-Poisson variation in log-linear models. Appl. Stat. 33, 38-44.

Di Fabio, R. (1987). Reliability of computerized surface electromyography for determining the onset of muscle activity. Phys. Ther. 67, 43-48.

Durgin, F. H., Evans, L., Dunphy, N., Klostermann, S., and Simmons, K. (2007). Rubber hands feel the touch of light. Psychol. Sci. 18, 152-157.

Ehrsson, H. H. (2007). The experimental induction of out-of-body experiences Science 317, 1048.

Ehrsson, H. H., Holmes, N. P., and Passingham, R. E. (2005). Touching a rubber hand: feeling of body ownership is associated with activity in multisensory brain areas. J. Neurosci. 25, 10564-10573.

Ehrsson, H. H., Spence, C., and Passingham, R. E. (2004). That's my hand Activity in premotor cortex reflects feeling of ownership of a limb. Science 305, 875-877.

Hodges, P., and Bui, B. (1996). A comparison of computer-based methods for the determination of onset of muscle contraction using electromyography. Electroencephalogr. Clin. Neurophysiol/Electromyogr. Motor C. 101 511-519.
Ijsselsteijn, W., de Kort, Y., and Haans, A. (2006). Is this my hand I see before me? The rubber hand illusion in reality, virtual reality and mixed reality. PresenceTeleop. Virt. 15, 455-464.

Lenggenhager, B., Tadi, T., Metzinger, T., and Blanke, O. (2007). Video ergo sum: manipulating bodily self-consciousness. Science 317, 1096-1099.

McCullagh, P., and Nelder, J. A. (1989). Generalized linear models. New York, Chapman and Hall.

Pavani, F., Spence, C., and Driver, J. (2000). Visual capture of touch: out-of-thebody experiences with rubber gloves. Psychol. Sci. 11, 353-359.

Pfurtscheller, G., Leeb, R., Keinrath, C., Friedman, D., Neuper, C., Guger, C., and Slater, M. (2006). Walking from thought. Brain Res. 1071, 145-152.

Sanchez-Vives, M. V., and Slater, M. (2005). From presence to consciousness through virtual reality. Nat. Rev. Neurosci. 6, 332-339.

Slater, M. (2004). How colorful was your day? Why questionnaires cannot assess presence in virtual environments. Presence-Teleop. Virt. 13, 484-493.

Tsakiris, M., and Haggard, P. (2005). The rubber hand illusion revisited: visuotactile integration and self-attribution. J. Exp. Psychol. Hum. Percept. Perform. $31,80-91$. 